\title{
Electronic medical records and NDM in U.S. emergency departments: A preliminary analysis
}

\author{
Markus A. Feufel \\ Wright State University \\ feufel.2@wright.edu
}

\author{
Valerie L. Shalin \\ Wright State University \\ valerie.shalin@wright.edu
}

\begin{abstract}
Motivation - Electronic medical records (EMR) have been hailed for their potential benefits. As EMR technology begins to be implemented, this research investigates its impact on emergency medicine. Research approach - 150 hours of naturalistic observations of emergency physicians in two Midwestern hospitals were performed. Findings - Results suggest that for EMR benefits to emerge, results must be easily distributed and located, data entry formats must support both input and later review, formatting must reveal changes over time for quantitative data, and records must support administrative and logistic functions. Research limitations - We cannot quantify the prevalence or cost of the current limitations in EMR. Originality/Value - This research is a first investigation of the impact of EMR systems on NDM in the field. Based on preliminary analyses, implications for (re)design of EMR systems are provided. Take-away message - To support practitioners, EMR systems must be grounded in a better understanding of the basic functions emergency physicians need to satisfy.
\end{abstract}

\section{Keywords}

Electronic medical records, emergency medicine, computer support systems, medical culture in the U.S.

\section{INTRODUCTION}

One major challenge for U.S. emergency departments (ED) is the fragmented nature of the U.S. emergency system (IOM, 2006). Multiple stakeholders are involved in emergency care delivery such as 911 call centers, Emergency Medical Services (EMS), ED physicians, family doctors, and consultant specialists. Though trauma systems have been established to improve the regional organization of emergency care (NHTSA, 2007), there is evidence that coordination across multiple providers is still suboptimal (Branas, MacKenzie, Williams, Schwab, Teter et al., 2005), seldom standardized (IOM, 2006), and could benefit from interventions that bridge the gap between multiple providers.

At the same time, ED residents are taught at the beginning of their residency that up to $80 \%$ of the diagnosis may be made based on a patient's previous medical history (PMH). The remaining 20\% come from current clinical measures and the history of present illness (HOPI). Yet ED physicians are mostly unfamiliar with their patients. Moreover, ED patients are sometimes quite ill and consequently 'poor historians' who can provide the physician neither with PMH nor an adequate HOPI. Some patients may be ashamed of (e.g., in case of a patient who contracted a sexually transmitted disease) or, in some cases, even try to conceal the actual reasons for their ED visit (e.g., in order to get pain medication, a 'drug seeking' patient may present with a bogus medical complaint). A patient's PMH reveals why, where, and when a patient has obtained care in the past as context for the interpretation of a patient's current clinical complaint. Ideally, the lab work and interventions resulting from the current ED visit appear in the PMH for the next healthcare provider.

Electronic medical records (EMR) promise better information integration and sharing across multiple providers based on better record accessibility, support for care continuity, outcome management, and process automation. Apart from problems of data confidentiality (e.g., Mandl, Szolovits, \& Kohane, 2001) and effective implementation of EMR into existing systems (e.g., McDonald, 1997), it is largely unclear in what way EMR change current work practices in the field. This paper will provide a first analysis of how EMR system impact NDM in the ED and make grounded suggestion of how future EMR systems can be improved to help providers meet the growing demand of emergency care in the U.S. (cf. NCHS, 2007).

For this paper, we consider the current and potential future implications of EMR in relation to the primary constraints on ED medicine: The need to provide clinical care (i.e., evaluations, treatments, and dispositions) in a timely and medically adequate manner, prevailing legal regulations that mandate medical care to every ED patient (Bitterman, 2000), and economic pressures from a growing number of patients, many without medical insurance (NCHS, 2007) or primary care providers (see Shi \& Singh, 2008). 


\section{METHOD}

About 150 hours of observations of (resident and attending) ED physicians provide a resource for identifying every-day patient management strategies in U.S. American EDs. Observations took place in two Midwestern EDs. The hospitals housing the EDs are both teaching hospitals affiliated with the medical school of a Midwestern public university. The EDs were selected because they are staffed by the same physician group (i.e., the same group of doctors) but differ along several organizational dimensions and with respect to characteristics of the patient populations they serve. Specifically with respect to the patient population, ED1 is frequented by patients who tend to be African American, without insurance, and suffer from life-threatening medical conditions such as organ failures or drug overdoses. The typical patients frequenting ED2 tend to be geriatric, Caucasian, middle-class, insured Americans who present with complaints such as chest pain or shortness of breath. Both EDs have a sizable population of urgent care patients who present with minor medical problems.

With respect to the technological support available at the observed EDs, physicians' and nurses' stations at both EDs are equipped with computer work stations. Only ED1 provides computers in the treatment rooms. ED1 has recently started to use an EMR and charting system. ED2 relies on a paper-based charting system using triage sheets (t-sheets) for documentation of patient's complaints and medical decision making but uses an EMR system for display and storage of lab results and medical imaging. Thus, the comparison of the selected EDs is particularly suited to investigate the impact of computer use on ED practice in two very different settings while simultaneously controlling for staff differences.

\section{RESULTS AND DISCUSSION}

We present and discuss observations in five areas: accessing clinical data, entering and requesting clinical data, reading/integrating clinical data, billing/legal functions and patient logistics. We contrast staff behavior with and without EMR, noting both benefits and limitations of the current EMR systems. Key observations appear in italics at the beginning of each subsection, along with abbreviated observations integrated in the text.

Accessing clinical data: Records must be easily distributed and located.

In ED2, where the t-sheet (paper based triage sheet) system is being used, we observed physicians, nurses, or assistant staff who spent considerable amounts of time searching for their patient's charts.

T-sheets are kept on a clip board (while in use) or in a file cabinet and can only be used by one provider at a time, creating a chart availability problem. Once filed in cabinets, t-sheets must be retrieved manually by already busy medical staff. During our observations, only a few incidences have been observed where practitioners ordered t-sheets from a different institution, exchanged via fax. Handwritten notes in constrained spaces required the recipient to decipher the previous physician's handwriting. The alternative is to identify, contact or page, and wait for a response from a patient's previous care provider. Yet in this case, ED physicians spend much time explaining the patient's current complaint before they get information that is useful for the case at hand.

EMR potentially avoid these difficulties of information retrieval. Because data entry and access is mostly done online, multiple providers (e.g., attending and resident physicians or offsite physicians) have immediate and simultaneous access to patients' charts. Yet current EMR systems are far from ideal and just like every new technology, EMR introduce new breakpoints into the workflow of ED physicians (cf. the envisioned world problem by Woods \& Dekker, 2000). EMRs don't work well if they are isolated. Suboptimal communication between disparate electronic record systems (within and between institutions) and the persistence of paper-based records prevent EMR from realizing their full potential. For example, we observed that non-existent communication between systems used by cardiology and ED nurses required ED nurses to transcribe data by hand from one system and enter it into the other to make information available in a format that can be used by nurses on the cardiology ward.

Entering and requesting clinical data: Data entry must support both input and comprehension and identify the author.

[Resident is charting for an asthma patient]: patient is short of breath with a history of asthma, 2 days ago patient had an asthma flair and tightness sensation; Albuterol ${ }^{l}$ didn't help; patient is semi-compliant: patient is supposed to take Advair ${ }^{2}$ b.i.d. [twice a day] but took it only once; no fever or chills, no intubation history; patient is a smoker; was advised to stop; pregnancy test was done even though patient had hysterectomy, patient didn't tell; obese abdomen; normal chest x-ray; no PE [pulmonary embolism] evidence from history or exam.

\footnotetext{
${ }^{1}$ Albuterol is a bronchodilator that relaxes muscles in the airways and increases air flow to the lungs (see http://www.drugs.com/albuterol.html).

${ }^{2}$ Advair is a medication used to prevent asthma attacks (see http://www.drugs.com/advair.html).
} 
Paper-based t-sheets have a number of advantages. They provide complaint-relevant check-boxes to (remind providers to) collect pertinent patient data and HOPI. The check-box style is judged a convenient and time-saving feature by most physicians. T-sheets also provide images of complaint-relevant body parts. That is, $\mathrm{t}$-sheets permit alphanumeric input while enhancing the understanding of a patient's complaint with visual representations. However, because they are preprinted, t-sheets provide, if at all, limited space for notes. For example, during one incident we observed an attending physician instructing her resident about the importance of time-stamping when she paged the hospital's stroke team ${ }^{3}$. The attending had to squeeze in the time stamp in tiny print to fit it between multiple medication orders because the tsheet did not offer space for the time stamp. Another potential drawback of checklists is that they may lead physician to mindlessly check off values without assessing the situation-specific needs and concerns of a particular patient.

The space constraints for charts in EMR systems are less strict as the charts are laid out as running text with a few subsection that correspond to HOPI, PMH, EKG/x-ray readings, and medical decision making. Thus, additional text can always be included by adding another line. Electronic charts are therefore less structured (compared to the check-box format described above), and less visually compelling in that text is the only input possible. From our observations (including the one at the beginning of this subsection), it became obvious that typing/dictating results in more elaborate formulations (more revisions) and sentence structures than handwritten charts as well as longer paragraphs because electronic charts provide unlimited space for text. Review of these charts will likely take longer because they require selective text scanning.

While EMR systems allow data access, entry, and test orders to be performed at any work station that is connected to the system, most physicians we observed accessed and entered clinical data at their designated work station after they had talked to the patient. Only one physician accessed and typed in clinical data while interviewing the patient. This may be due to the fact that physicians who grew up with the t-sheet system are used to doing chart work at the physicians' station. An alternative explanation is that the computer workstations are not oriented towards the patient but towards the wall, so that typing requires the physician to turn away from the patient, which may appear rude or inattentive. Moreover, EMR access in ED1 is currently constrained by the fact that work stations have recently been equipped with a dictating device that can be used to verbally input patient data. This requires physicians to provide a 15-minute speech sample (for each work station) so that the dictating software recognizes the physician's intonation. Most physicians provide a speech sample only once and are consequently 'tied' to this particular station. Although dictation is far from being perfect and translation errors are common, most junior physicians like the new features as they perceive it to be faster than typing. The majority of senior physicians are not used to dictating and feel that typing the report is not only more comfortable but also faster. Physicians therefore require alternative input modalities.

The availability of multiple modalities becomes particularly important if one modality breaks down or is error-prone. For example, the job of ED admission nurses is to collect data on patients who will be admitted to the hospital, such as diet and medication requirements or insurance information. To do this job efficiently, nurses have been provided with laptops (which are connected to the hospital's wireless network) so that they can take in the data while talking to the patient. However, nurses do not use the laptops for its intended purpose because "they freeze up easily and the wireless connection does not work in the rooms." Instead, the nurses developed their own work sheet to query the patient and take notes. They enter obtained data after the interview standing on the hallway where the wireless connection of the laptops is better. This is still not optimal, the nurse said, "if the computer freezes up or the wireless connection breaks down, all entered but not saved data is gone, it's difficult to get through one sitting." In both EDs, we have observed computer breakdowns causing delays of physicians' work procedures. Whereas the observed incidences were caused by the malfunctioning of single work stations and had minor consequences, an EMR system breakdown - e.g., if too many people access and use similar system components at the same time - may have far more serious consequences for overall ED functioning.

The brittleness of computer technology speaks to the need of back-up and surrogate documentation systems. In ED1, the EMR became a mechanism for issuing orders, (via the nurses who would get the specimens and administer medications) and paged consultants (via two secretaries who would make the actual page).To order medical tests, physicians in ED1 had to first select tests and then sign the orders using a fingerprint reader. However, one physician had difficulties (requiring up to 5 attempts) signing his orders because the reader did not recognize his fingerprints. To circumvent the fingerprint reader, the physician had to click through multiple menus before the order could be placed. In sum, to increase the resilience and usability of EMR systems, future systems need to provide multiple input modalities that can be used interchangeably according to individual preferences but maintain full functionality if one function breaks down.

\footnotetext{
${ }^{3}$ The effective treatment of strokes with TPA (tissue plasminogen activator is a substance that quickly breaks down blood clots) can only be performed within the first three hours after a stroke
} 
In some cases, a provider (e.g., resident/attending) made an order whereas another provider deleted it. One category of deletion was due to lab technicians deleting an order, which got entered twice ${ }^{4}$. In one incidence, a nurse came by the resident's desk to ask about an order of a d-dimer ${ }^{5}$ because it 'looks like it was discontinued.' After checking in the system the resident speculates: "It doesn't say who discontinued it. Looks like a repeat d-dimer order and they [the lab] canceled it $^{6}$ but anyway, another d-dimer is still on the order list." The other category was due to supervisors (the attending physician) adding or deleting orders that have been made by residents. However, at the time the system did not provide information as to who and why the order got deleted. In both cases, the primary providers had to find out the reason for the deletion before they could reorder the test. (One might argue that the EMR actually made the conflict apparent.) Although the EMR system logs who does what and when, the problem was that data and actions were not tagged with an author identifier ${ }^{7}$. The fact that computer displays are mainly concerned with the state of the controlled system but less so with the identity of the controller seems to be a general problem for computer supported coordination of work processes across multiple providers (see Shalin \& McCraw, 2003 for an example in space operations). Effective use of EMR systems requires that information about an action and the action's author be easily available upon request and that changes made to an existing order be matched with a rationale.

Reading/integrating clinical data: Formatting must reveal changes over time for quantitative data.

Attending: (re pt in 47) you checked all the boxes[ordered all basic labs] for her...

Resident: she had a gap of $20^{8}$.

Attending: but she has been running low anyway... look at it.

One advantage of EMR is that data in electronic format can be reformatted easily. During the observations, physicians in both EDs took advantage of EMR display features that allowed viewing and comparing x-rays and cat scans with images that had been taken during a previous visit. This feature allows ED physicians to easily differentiate out-of-thenorm developments from chronic disease, a distinction that is essential for the practice of emergency medicine. In ED1, the same procedure could be used to display basic lab values. However, time series of pertinent values are currently represented by numbers in tables and require additional clicking to display. Arrows next to lab values indicate values above/high (H) or below/low (L) the normal/average range. For patients with long PMH, this table format often did not fit on one screen (both EDs use 17" screens) and required scrolling, which made the interpretation of the data more difficult. Moreover, the patient's average range on a certain lab value is currently not displayed but has to be inferred. The limitation of this data format represented a particular disadvantage for junior residents. During our observations, these residents often missed long-term, chronic developments. As the above example illustrates, the attending physicians noted that the obtained values did not require immediate treatment in the ED but could be managed by discharging the patient and making him or her follow up with a specialist.

In sum, future EMR systems should incorporate the feature of displaying lab values as simple graphs of time series that indicate normal as well as patient's average values. For example, Tufte (1992) has shown that intelligent information representations can help to condense complex data into handy time series that can be inserted in text (e.g., the patient's chart) or stand alone during the physician's review of lab results. This simple reformatting could not only save time intensive clicking and menu navigation but also help doctors easily recognize out-of-the-norm values as well as developments over time. As of now, ED physicians must transform essential information in their heads requiring resources that could be put to better use elsewhere.

EMR and billing/legal function: Medical records must support administrative functions.

Attending (comes by the Resident's desk): Your charts are fine except we are not getting the billing pieces for $E K G$ s and $x$-ray readings; if it is read by the EDP [ED physician] we get paid, if it is read by the radiologist they get paid; if you use the template in EPIC [the EMR system] (the Attending shows the Resident how to enter the template) it will give you all the elements to make sure we get paid; you have all the important

\footnotetext{
${ }^{4}$ It is customary, however, to order repeat enzymes as they show heart problems only over time

5 D-dimer tests are ordered to help rule out, diagnose, and monitor diseases related to blood clots. (see http://www.labtestsonline.org/understanding/analytes/d_dimer/test.html)

${ }^{6}$ Medically, it does not make sense to order two d-dimers for a patient during one visit.

${ }^{7}$ Note that handwriting can serve this function in t-sheets.

${ }^{8}$ The anion gap measures the difference between anions and cations in a person's blood. This value indicates acid-base imbalances in the body such as metabolic acidosis. (see http://www.labtestsonline.org/understanding/analytes/electrolytes/faq.html)
} 
medical information in your chart but not the ones for billing; we get $\$ 15-16$ for an EKG reading and $\$ 25$ for an $x$-ray reading.

ED physicians must satisfy strict billing and legal requirements. Documentation of how these requirements were satisfied is essential for reimbursement and the prevention of malpractice lawsuits. The above observation explains the importance of this function for a new resident. Thus, patients charts, be it t-sheets or EMR, contain potentially distracting data that are not immediately relevant to the clinical function of emergency care. EMR systems could make the same information available in different views, so that pertinent information is provided depending on the function (e.g., the clinical, the billing/legal, or the logistic function) that is most salient to emergency physicians at a given time.

\section{EMR and patient logistics}

Resident: Let's go check on our belly pain lady in the hallway. [when standing in front of an empty bed] where did she go [to some of the nurses] what did you with my belly pain patient?

Nurse: I think Sharon put her in 24, let me go ask her.

As has been shown for space shuttle controllers (Shalin \& McCraw, 2003), efficient work coordination requires information regarding the system status as well as the current activities of fellow workers. Similarly, ED care planning may be improved by supplementing EMR system with logistic information. The function of patient logistics is only rudimentarily considered in the design of current EMR system. For example, early expert treatment has been shown to be related to more favorable clinical outcomes (e.g., Diercks, Roe, Chen, Peacock, Kirk, et al., 2007; Hollander \& Pines, 2007). To achieve early inclusion of experts in the treatment process requires efficient communication across hospital wards. However, given an increasing difficulty of finding on-call consultants for ED patients (IOM, 2006), our observations have shown that it is quite common for ED physicians to engage in lengthy phone calls and discussions trying to convince consultants to admit patients who cannot receive adequate treatment in the ED. This procedure is time consuming, costing physical and cognitive resources.

The logistics problem is acute in the case of patient transfer to another hospital, sometimes the result of a patient's PCP or specialty provider. Typically, the PCP's or specialty provider's name is, if at all, provided by the patient or his/her family. The phone number must be looked up in a database, which is not integrated with the EMR system used by either ED. In the absence of direct provider contact, physicians and/or ED secretaries must search in folders or on notes that are pinned to the walls in the provider station. According to some of the observed physicians, this data changes rapidly so that a computerized version, which can be accessed by the providers themselves, would reduce the work of maintaining the folder and, more importantly, searching for the relevant information. Provider's preferences could be associated with a particular patient so that the provider can be immediately paged. In addition, each hospitalist or admitting group of physicians has particular admission preferences. This information is known by experienced physicians but less so by junior attending and resident physicians. Short notes of provider preferences could make this information available to all ED practitioners and accelerate admission processes.

Based on our observations (see above example), confusion and suboptimal coordination of work procedures has occurred because doctors were unaware of bed management in the ED (managed by the nurses but often not communicated to the doctors) and/or the status and location of individual patients (e.g., the patient has been moved to a different room or is currently in the cat scanner). On the other hand, patient status information, which is currently provided by the EMR system in ED1 (e.g., informing the doctor that the patient is waiting for a provider, waiting for discharge, or waiting for an inpatient bed), was frequently used by the observed ED physician to sort, prioritize, and effectively manage their work procedures. Thus, logistic information may help ED physicians to improve information and resource management, ultimately optimizing ED utilization and seamless, quality care from ED triage to hospital discharge (e.g., Aronsky, Jones, Lanaghan, Slovis, \& Corey, 2008).

\section{CONCLUSION}

This paper has provided a preliminary analysis of the benefits and drawbacks of EMR and their implication for NDM in emergency medicine. The assumption underlying our analysis is that, to be successful, EMR must support the multiple functions ED practitioners have to satisfy. For this paper, we discussed the implications of EMR for NDM with respect to three major functions of emergency medicine, the clinical, billing/legal, and logistic function. Based on this analysis four major issues of EMR in NDM can be summarized. First, the full potential of EMR can only be exploited once current documentation systems become more integrated and communication across and within EMR systems is better supported (see McDonald, 1997). That is, even if single hospitals or hospital groups develop their own EMR system, the full advantage can only occur when systems and data formats facilitate communication across the larger health care system including patient's PCPs, private practices, and competing hospitals. Second, to improve the usefulness of EMR system, it will be essential to create data representations and formats that explicitly model and state the functions served by the EMR for a specific user group (e.g., ED physicians versus cardiologists) as well as the documentation therein (e.g., billing/legal versus clinical purposes). Third, to be able to maintain ED resilience, the brittleness of computer technology requires redundant use of hard and software features such as redundant data input and access modalities (cf. 
Hollnagel, Woods, \& Levenson, 2006). Finally, we consider the support of the logistic function worthy of a separate category because it has so far been largely neglected in the design of EMR system but plays an essential role in managing increasing patient volumes and ultimately, creating disaster preparedness. Although different documentation systems have been observed, more studies must be performed across the U.S. and with different EMR systems to quantify the prevalence and cost of the current limitations in EMR.

\section{REFERENCES}

Aronsky, D., Jones, I., Lanaghan, K., \& Slovis, C. (2008). Supporting patient care in the emergency department with a computerized whiteboard system. Journal of the American Medical Informatics Association, 15(2), $184-194$.

Bitterman, R. A. (2000). Providing emergency care under the federal law: EMTALA. Dallas, Texas: American College of Emergency Physicians. Branas, C. C., MacKenzie, E. J., Williams, J. C. Schwab, C. W., Teter, H. M., Flanigan, M. C., et al., (2005). Access to Trauma Centers in the United States. The Journal of the American Medical Association, 293(21), 2626-2633.

Diercks, D., Roe, M., Chen, A., Peacock, W., Kirk, J., Pollack Jr., C., et al. (2007). Prolonged Emergency Department stays of non-ST-segment-elevation myocardial infarction patients are associated with worse adherence to the American College of Cardiology/American Heart Association guidelines for management and increased adverse events. Annals of Emergency Medicine, 50(5), 489-496.

Hollander, J. \& Pines, J. (2007). The Emergency Department crowding paradox: The longer you stay, the less care you get. Annals of Emergency Medicine, 50(5), 497-499.

Hollnagel, E., Woods, D. D., \& Leveson, N. (2006). Resilience engineering: Concepts and percepts. Burlington, VA: Ashgate Publishing.

IOM (2006). The future of emergency care in the United States health system. Retrieved April 17, 2008, from Institute of Medicine Web site: http://www.iom.edu/Object.File/Master/35/014/Emergency\%20Care.pdf

Mandl, K., Szolovits, P., \& Kohane, I. S. (2001). Public standards and patients' control: how to keep electronic medical records accessible but private. BMJ: British Medical Journal, 322(7281), 283.

McDonald, C. J. (1997). The Barriers to Electronic Medical Record Systems and How to Overcome Them, J Am Med Inform Assoc. 4(3), 213-221.

NCHS (2007). National Hospital Ambulatory Medical Care Survey: 2005 Emergency Department Summary, Tables 1, 11, 13, 22, 25. Retrieved December 15, 2007, from National Centre for Health Statistics (NCHS): Emergency department visits Web site: http://www.cdc.gov/nchs/fastats/ervisits.htm

NHTSA (2007). Trauma systems: Agenda for the future. Retrieved December 15, 2007, from NHTSA Web site: http://www.nhtsa.dot.gov/people/injury/ems/emstraumasystem03/index.htm

Shalin, V. L. \& McCraw, P. M. (2003). Representations for distributed planning. In E. Hollnagel (ed.) Handbook of cognitive task design. (pp. 701-725) New Jersey: Lawrence Erlbaum Associates.

Shi, L. \& Singh, D. A. (2008). Delivering Health Care in America: A Systems Approach. (5th Edition) . Subury : Jones and Bartlett Publishers.

Tufte, E. R. (1992). The visual display of quantitative information. Los Angeles, CA: Graphic Press.

Woods, D. D. \& Dekker, S. (2000). Anticipating the effects of technological change: A new era of dynamics for human factors. Theoretical Issues in Ergonomics Science, 1, 3, 272-282. 